Original Paper

\title{
Polyimide Filaments as a Novel Stationary Phase in Packed-Capillary Gas Chromatography
}

\author{
Koki NAKAGAMI ${ }^{1}$, Ohjiro SUMIYA ${ }^{1}$, Toshiaki TAZAWA ${ }^{1}$, Tomoya MONOBE ${ }^{1}$, \\ Mitsuru WATANABE ${ }^{1}$, Ikuo UETA², Yoshihiro SAITO*1 \\ ${ }^{1}$ Department of Environmental and Life Sciences, Toyohashi University of Technology, 1-1 Hibarigaoka, Tempaku-cho, \\ Toyohashi 441-8580, Japan \\ ${ }^{2}$ Department of Applied Chemistry, University of Yamanashi, 4-3-11 Takeda, Kofu 400-8511, Japan
}

\begin{abstract}
A fine fibrous polyimide (PI) material was introduced as a stationary phase in packed capillary gas chromatography (GC). Packed longitudinally into a capillary, the bundle of the PI filaments exhibited retentivity for a set of test analytes, showing that the PI-packed column was able to work as a stationary phase in GC. On the basis of a systematic consideration of the retention factors for homologous alkanes, it was confirmed that a good linear relationship between the carbon number and the logarithmic retention factor for alkanes at a constant column temperature. Linear van't Hoff plots were also obtained for alkanes, where logarithmic retention factors were plotted against the reciprocal absolute column temperatures. On the basis of the tendency in the retention factors of homologous alkane test analytes, the PI stationary phase has a trend for the retention behavior similar to that of conventional GC stationary phases widely employed, although a certain contribution of polar functional groups in the PI stationary phase to the retention of alcohols was observed. Taking advantage of the good heat-resistance of the PI material, an applicability to temperature-programmed GC separations was also suggested for the fibrous PI stationary phase.
\end{abstract}

Keywords: Polyimide; Fiber; Stationary phase; Packed-capillary column; Alkanes; van't Hoff plot; Retention behavior; Gas chromatography; Temperature program

\section{Introduction}

Gas chromatography (GC) is one of the most powerful and popular techniques for the separation of volatile organic compounds. In addition to the development of $\mathrm{GC}$ instruments including the specially-designed detectors and injectors, various types of stationary phases have been developed and commercialized for an effective separation of the complex mixtures in GC [1-3]. Miniaturization of analytical systems is another approach to realize a more effective separation, where the down-sizing of the separation system have been studied along with an appropriate coupling to modern sample preparation techniques [4-14].

In order to develop novel separation methods and

${ }^{*}$ Corresponding author: Yoshihiro SAITO

Tel: +81-532-44-6803; Fax: +81-532-48-5833

E-mail: saito@ens.tut.ac.jp corresponding appropriate sample preparation techniques, various types of fibrous polymeric materials have been introduced [13-35]. A bundle of fine fibrous materials was packed into a short extraction cartridge for the sample preparation of gaseous [13-18] and liquid [19-35] sample matrices. The use of the fibrous materials as the stationary phase was investigated in chromatographic techniques including GC [36-42], capillary electrochromatography [43-45] and liquid chromatography [46-52]. Fiber-packed capillary was also studied as a miniaturized interface between two-dimensional separations $[53,54]$.

Taking advantage of a good heat-resistance, several synthetic polymer filaments have been employed as a stationary phase for high temperature separations in GC,

Received: 6 April 2018

Accepted: 21 April 2018

J-STAGE Advance Published: 29 April 2018

DOI: $10.15583 /$ jpchrom.2018.008 
while the sample preparation of liquid samples was carried out with the mini-cartridge packed with a bundle of these filaments. This is because the fibrous material having a good heat-resistance also have a good solvent-resistance that is normally required in the conventional sample preparation procedure [32-37].

A novel polyimide (PI) particle-packed capillary was successfully introduced as the sample preparation medium for gaseous samples in the previous work [55], suggesting a possibility of a PI-based material to the applications to a novel stationary phase in chromatographic separations. As an extension of the previous investigation, in this work, a fine fibrous PI material was introduced as a novel stationary phase in packed-capillary GC. Since the PI fiber has carbonyl functional groups in the structure, a unique selectivity can be expected on the basis of the chemical structure and the resulting ordering of the polymer chains. For the evaluation of the retention behavior of the fibrous PI stationary phase, a set of test analytes including homologous alkanes and the corresponding alcohols were employed.

\section{Experimental}

\subsection{Reagents and sample analytes}

All of the reagents and solvents were of analytical reagent grade. All the alkanes and the corresponding alcohols were obtained from Tokyo Chemical Industry (Tokyo, Japan), and hexane was obtained from Kishida Chemical (Osaka, Japan). For the evaluation of the retentivity, a set of analytes consisted of benzene, toluene, ethylbenzene, $o$-xylene, $m$-xylene and $p$-xylene (i.e. BTEX) was obtained from Wako Pure Chemical (Osaka, Japan).

\subsection{Preparation of the packed capillary column}

As the fibrous PI stationary phase, a bundle of P84 filaments [56] was obtained from Toyobo, Otsu, Japan. The chemical structure of P84 is illustrated in Fig. 1. Detail of the PI material can be found elsewhere [56]. For the preparation of the capillary column, a bundle of precounted P84 filaments (consisted of 160 filaments) was packed into a fused-silica capillary of $0.32 \mathrm{~mm}$ i.d., $1.0 \mathrm{~m}$ length (Shinwa Chemical Industries, Kyoto, Japan). The P84 filaments have an average cross-section area of $c a$. 1.6 $\times 10^{-4} \mathrm{~mm}^{2}$, corresponds a diameter of $c a .14 .3 \mu \mathrm{m}$ if a perfectly circular cross-section is assumed. The packing density on the basis of the total cross-section area of the filaments to that of the capillary opening was calculated as about $32 \%$.

To ensure the parallel alignment of all P84 filaments in the capillary, the packing process was carried out in a similar manner as described previously [28]. First, an appropriate length of poly(vinylidene fluoride) (PVF) fishing line (64 $\mu \mathrm{m}$ o.d., Kureha, Tokyo, Japan) was inserted as a guide into the capillary. The end of the PVF guide fiber has an extra length to form a loop outside of the capillary, and then, second PVF fiber is inserted into the loop of the first guide fiber, and next, the first guide fiber is pulled from the other end of the capillary. The bundle of precounted P84 filaments (with a pre-cut length of $c a$. 2.4 $\mathrm{m}$ for a $1.0-\mathrm{m}$ packed section) to be packed is inserted into the loop of the second guide fiber, where the front-end of the bundle should be appropriately bent to make sure a smooth introduction into the capillary. The second PVF guide fiber is pulled from the other side of the capillary carefully to produce uniform introduction of the bundle into the capillary. Finally, the second guide fiber is pulled out the capillary with care, and then cut the extra length of the filaments to prepare a packed-capillary column.

\subsection{GC measurements}

A 6890N gas chromatograph (Agilent, Santa Clara, CA, USA) with a split/split-less injection port and a frame ionization detector (FID) was used for all the GC measurements. All the measurements were carried out by a split mode with a typical ratio of 50:1. Injector temperature and detector temperature were typically set at $300^{\circ} \mathrm{C}$. $\quad \mathrm{N}_{2}$ was used as the carrier gas.

Before the evaluation of the retention behavior, the PI fiber-packed column was subjected to an appropriate preconditioning. Chromatogram was recorded with ChromNAV Chromatography Data Handling Software (Jasco, Tokyo, Japan) running on a personal computer.

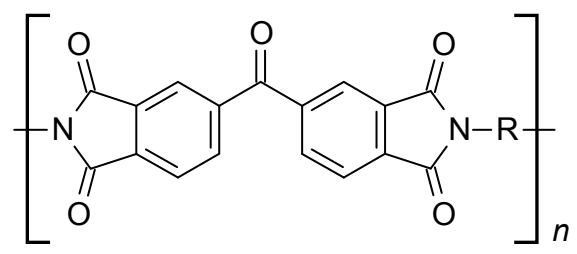<smiles>[R]=[Fe]Cc1cccc(C)c1C</smiles>

Fig. 1. Chemical structure of P84 PI [56].

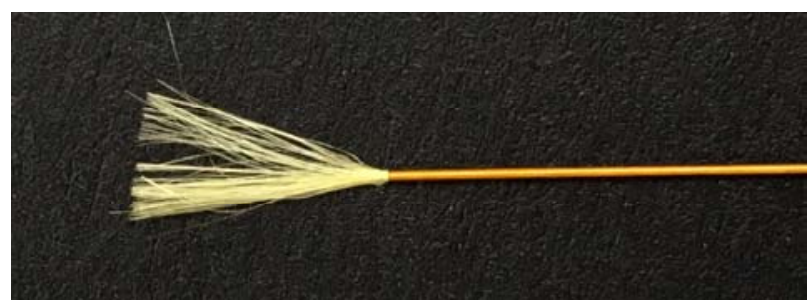

Fig. 2. Typical photograph of PI fiber-packed capillary column. 


\section{Results and discussion}

3.1. Retention of homologous series of alkanes on the fibrous PI stationary phase

Retention behavior of a set of homologous alkanes on the fibrous PI stationary phase was evaluated, where some fundamental experimental parameters, such as column head pressure, split ratio, injection volume of the sample, were optimized in the preliminary experiments with the PI-packed column that had been subjected to an appropriate preconditioning process before use. Fig. 3 shows the trend for the retention of alkanes on the fibrous PI stationary phase. As can be seen from the plots, a good linear relationship was found between the logarithmic retention factor and the number of carbon atoms for all the alkanes. The linear correlation factors for these plots were larger than 0.99 .

This results have a good agreement with that obtained conventional non-polar GC stationary phases such as polydimethylsiloxane (PDMS). For the separation of alkanes on the fibrous PI stationary phase, one can expect a separation similar to that on the basis of a general selectivity of typical PDMS stationary phases. On the PI stationary phase, the separation of normal alkanes was mainly according to the number of carbon atoms in the analyte, although a certain contribution of the polar functionalities in the PI structure to the retention can be expected.

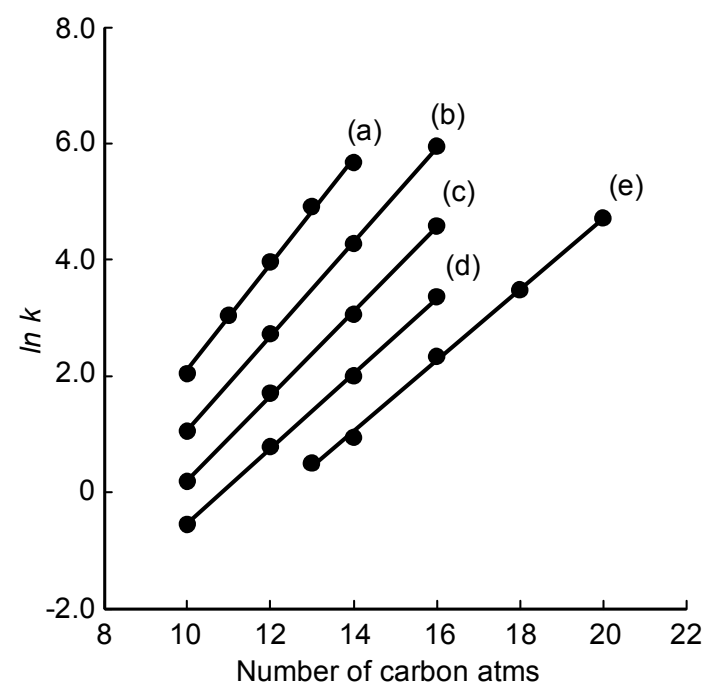

Fig. 3. Retentivity for normal alkanes having different carbon numbers at various column temperatures on the fibrous PI stationary phase. GC conditions; column head pressure, $100 \mathrm{kPa}$; injection mode, split; split ratio, 50:1. (a) $40^{\circ} \mathrm{C}$, (b) $60^{\circ} \mathrm{C}$, (c) $80^{\circ} \mathrm{C}$, (d) $100^{\circ} \mathrm{C}$ and (e) $120^{\circ} \mathrm{C}$. Other conditions are in the text.

3.2. van't Hoff plots of alkanes for the retention on the fibrous PI stationary phase

As one of the common approaches to the evaluation of new stationary phases, the logarithmic retention factor is plotted against the reciprocal absolute column temperature [57,58]. From the resulting van't Hoff plots, as typically shown in Fig. 4, a good linear relationship was confirmed for all the analytes, suggesting that the retention mechanism is consistent in the temperature range studied. The trend in the retention of homologous alkanes (Fig. 4) is quite similar to that observed on a conventional non-polar stationary phase in GC. The results demonstrated a possibility to an easy optimization approach to temperature-programmed separation on the PI stationary phase.

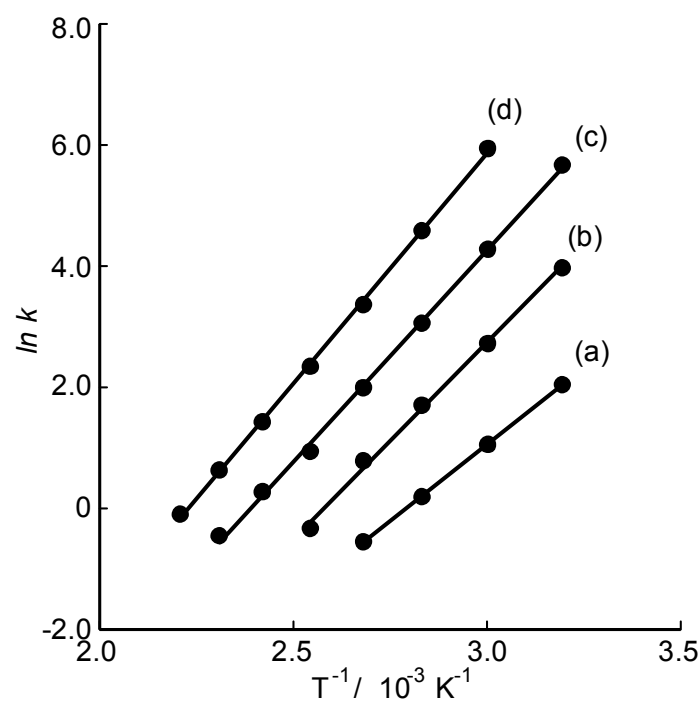

Fig. 4. van't Hoff plots for four alkanes on the PI stationary phase. (a) decane, (b) dodecane, (c) tetradecane and (d) hexadecane. GC conditions are the same as in Fig. 3.

Fig. 5 compares typical van't Hoff plots for two alkanes and the corresponding alcohols. As the same as alkanes, linear van't Hoff plots were also obtained for all the alcohols studied. However, the retention was much larger than that of the corresponding alkanes having the same number of carbons in their chemical structure. From the plots, the enthalpy of solute transfer from the carrier gas to the stationary phase was calculated as $-38.0,-42.0,-60.5$ and $-61.8 \mathrm{~kJ} / \mathrm{mol}$ for nonane, decane, 1-nonanol and 1-decanol, respectively. The contribution of the hydroxyl functional group in alcohols to the retention on the PI stationary phase is significantly larger than that of these alkane backbone, suggesting a contribution of a polar interaction between alcohols and the stationary phase.

\subsection{Selectivity for BTEX on the PI phase}

In order to further investigate the selectivity of the fibrous PI stationary phase, the retentivity of the PI phase for BTEX was compared with that obtained on a commercially available open-tubular DB-WAX capillary 
column $(0.25 \mathrm{~mm}$ i.d., $15 \mathrm{~m}$ length, $0.25 \mu \mathrm{m}$ film thickness; $\mathrm{J}$ and W Scientific, Folsom, CA, USA) as shown in Fig. 6. The selectivity of the fibrous PI phase to BTEX is quite similar to the selectivity obtained on the DB-WAX phase, i.e. a polyethyleneglycol (PEG) phase, especially for the elution order of three xylene isomers. The results also suggest a contribution of the polar moiety, carbonyl and imide groups, in the chemical structure of the PI stationary phase, although a further systematic investigation is necessary to derive the final conclusion for the retention mechanism from the retention data.

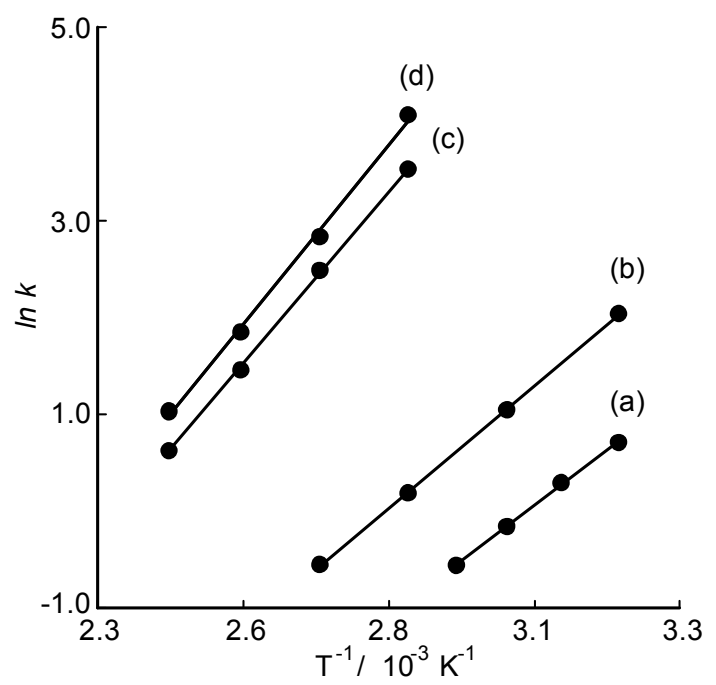

Fig. 5. Typical van't Hoff plots for alkanes and alcohols obtained on the PI stationary phase. (a) nonane, (b) decane, (c) 1-nonanol and (d) 1-decanol. GC conditions are the same as in Fig. 3.

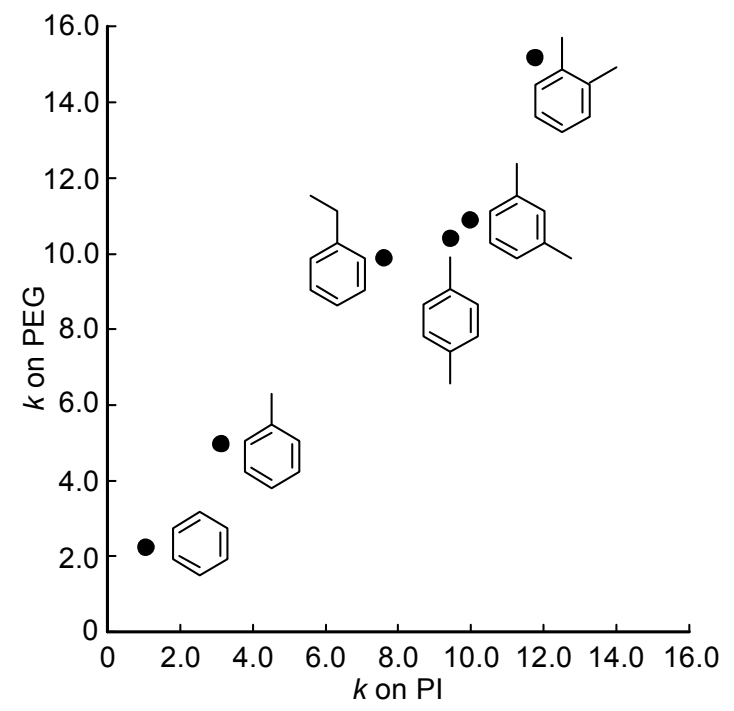

Fig. 6. Comparison of the retentivity for BTEX on the PI and DB-WAX (PEG) phases. GC conditions for DB-WAX phase: injector and detector temperature, $200^{\circ} \mathrm{C}$; column temperature, $30^{\circ} \mathrm{C}$. Other conditions are the same as in Fig. 3 .
3.4. Temperature-programmed separation on the PI fiber-packed capillary column

Taking advantage of the excellent heat-resistance of the PI filaments, a temperature-programmed separations was possible on the PI phase as typically shown in Fig. 7. As similar to the other fibrous stationary phases reported previously [36-38], the theoretical plate number was not well-comparable to a conventional open-tubular capillary column. This can be mainly due to the partial uniformity of the packed filaments in the capillary. However, the reproducibility in the column preparation is quite satisfactory on the basis of a careful packing process with a pre-counted number of filaments. In addition, the column was typically used for more than 100 times for the temperature-programmed separations up to $300^{\circ} \mathrm{C}$, where no significant loss in the retention performance was found.

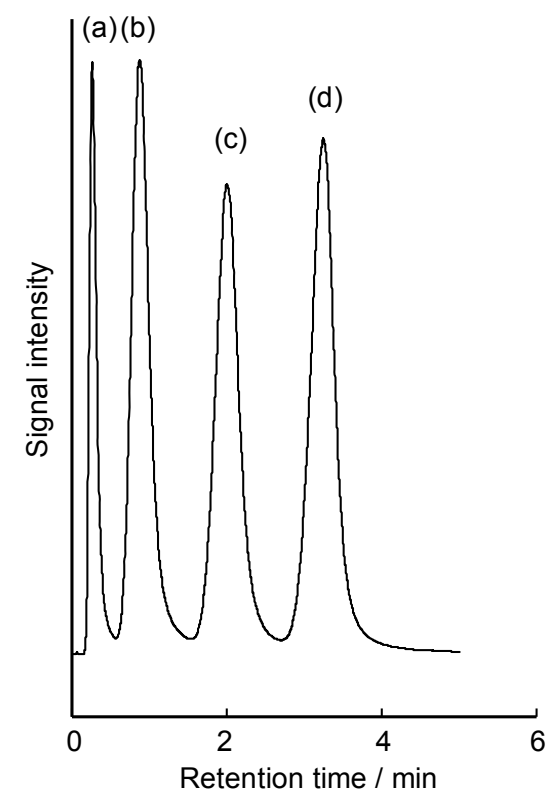

Fig. 7. Chromatograms for the separation of a homologous alkane mixture. Conditions: temperature program, $50^{\circ} \mathrm{C}$ to $150^{\circ} \mathrm{C}$ at the rate of $20^{\circ} \mathrm{C} / \mathrm{min}$. Peaks: (a) decane; (b) dodecane; (c) tetradecane and (d) hexadecane. Other conditions are the same as in Fig. 3.

\section{Conclusions}

In this work, a novel PI fiber-packed capillary column was introduced in GC. The results demonstrated a possibility of the PI phase as a stationary phase in GC. Because of a good heat-resistance of the PI filaments, temperature-programmed separations were possible on the PI phase.

In terms of the selectivity to analytes on the fibrous PI phase, there are only limited data at this stage, especially for the retention analysis of various types of polar compounds as the sample analytes. Therefore, a more 
systematic evaluation using various types of sample analytes having different polarities and chemical structures must be scheduled. These investigations are currently on going in our laboratory.

\section{Acknowledgements}

The authors would like to thank Mr. H. Sugiyama, Toyobo, for supplying the P84 PI filaments. The authors also thank Profs. A. Matsumoto and K. Takashima, Toyohashi University, for their helpful technical suggestions during this work. A part of this work has been financially supported as two KAKENHI projects (\#15K05537 and \#15K17875).

\section{References}

[1] Eiceman, G. A.; Gardea-Torresdey, J.; Dorman, F.; Overton, E.; Bhushan, A.; Dharmasena, H. P. Anal. Chem. 2006, 78, 3985-3996.

[2] Dorman, F. L.; Overton, E. B.; Whiting, J. J.; Cochran, J. W.; Gardea-Torresdey, J. Anal. Chem. 2008, 80, 4487-4497.

[3] Dorman, F. L.; Whiting, J. J.; Cochran, J. W.; Gardea-Torresdey, J. Anal. Chem. 2010, 82, 4775-4785.

[4] Saito, Y.; Jinno, K.; Greibrokk, T. J. Sep. Sci. 2004, 27, 1379-1390.

[5] Saito, Y. Chromatography 2003, 24, 7-17.

[6] Saito, Y.; Ueta, I. Chromatography 2017, 38, 85-94.

[7] Saito, Y.; Kawazoe, M.; Imaizumi, M.; Morishima, Y.; Nakao, Y.; Hatano, K.; Hayashida, M.; Jinno, K. Anal. Sci. 2002, 18, 7-17.

[8] Saito, Y.; Jinno, K. J. Chromatogr. A 2003, 1000, 53-67.

[9] Jinno, K.; Muramatsu, T.; Saito, Y.; Kiso, Y.; Magdic, S.; Pawliszyn, J. J. Chromatogr. A 1996, 754, 137-144.

[10] Saito, Y.; Kawazoe, M.; Hayashida, M.; Jinno, K. Analyst 2000, 125, 807-809.

[11] Inoue, M.; Saito, Y.; Ueta, I.; Miura, T.; Ohkita, H.; Fujimura, K.; Jinno, K. Anal. Sci. 2010, 26, 687-691.

[12] Ueta, I.; Takahashi, K.; Saito, Y. Anal. Sci. 2012, 28, 953-957.

[13] Ueta, I.; Saito, Y. Chromatography 2014, 35, 41-48.

[14] Ogawa, M.; Saito, Y.; Imaizumi, M.; Wada, H.; Jinno, K. Chromatographia 2006, 63, 459-463.

[15] Saito, Y.; Ueta, I.; Ogawa, M.; Jinno, K. Anal. Bioanal. Chem. 2006, 386, 725-732.

[16] Saito, Y.; Ueta, I.; Ogawa, M.; Hayashida, M.; Jinno, K. J. Pharm. Biomed. Anal. 2007, 44, 1-7.

[17] Ueta, I.; Saito, Y.; Ghani, N. B. A.; Ogawa, M.; Yogo, K.; Abe, A.; Shirai, S.; Jinno, K. J. Chromatogr. A 2009, 1216, 2848-2853.

[18] Nakagami, K.; Tazawa, T.; Sumiya, O.; Ueta, I.; Saito, Y. Chromatography 2018, 39, 75-81.
[19] Ueta, I.; Saito, Y. Bunseki Kagaku 2011, 60, 833-844.

[20] Ueta, I.; Saito, Y. Anal. Sci. 2014, 30, 105-110.

[21] Saito, Y.; Nakao, Y.; Imaizumi, M.; Takeichi, T.; Kiso, Y.; Jinno, K. Fresenius J. Anal. Chem. 2000, 368, 641-643.

[22] Imaizumi, M.; Saito, Y.; Hayashida, M.; Takeichi, T.; Wada, H.; Jinno, K. J. Pharm. Biomed. Anal. 2003, 30, 1801-1808.

[23] Jinno, K.; Kawazoe, M.; Saito, Y.; Takeichi, T.; Hayashida, M. Electrophoresis 2001, 22, 3785-3790.

[24] Saito, Y.; Imaizumi, M.; Takeichi, T.; Jinno, K. Anal. Bioanal. Chem. 2002, 372, 164-168.

[25] Saito, Y.; Nakao, Y.; Imaizumi, M.; Morishima, Y.; Kiso, Y.; Jinno, K. Anal. Bioanal. Chem. 2002, 373, 81-86.

[26] Saito, Y.; Jinno, K. Anal. Bioanal. Chem. 2002, 373, 325-331.

[27] Saito, Y.; Nojiri, M.; Imaizumi, M.; Nakao, Y.; Morishima, Y.; Kanehara, H.; Matsuura, H.; Kotera, K.; Wada, H.; Jinno, K. J. Chromatogr. A 2002, 975, 105-112.

[28] Saito, Y.; Imaizumi, M.; Ban, K.; Tahara, A.; Wada, H.; Jinno, K. J. Chromatogr. A 2004, 1025, $27-32$.

[29] Imaizumi, M.; Saito, Y.; Ban, K.; Wada, H.; Hayashida, M.; Jinno, K. Chromatographia 2004, 60, 619-623.

[30] Abe, A.; Saito, Y.; Imaizumi, M.; Ogawa, M.; Takeichi, T.; Jinno, K. J. Sep. Sci. 2005, 28, 2413-2418.

[31] Qi, D.; Kang, X.; Chen, L.; Zhang, Y.; Wei, H.; Gu, Z. Anal. Bioanal. Chem. 2008, 390, 929-938.

[32] Ogawa, M.; Saito, Y.; Shirai, S.; Kiso, Y.; Jinno, K. Chromatographia 2009, 69, 685-690.

[33] Nakane, K.; Tazawa, T.; Mori, Y.; Kobayashi, A.; Ueta, I.; Saito, Y. Chromatography 2015, 36, 61-65.

[34] Ogawa, M.; Saito, Y.; Ueta, I.; Jinno, K. Anal. Bioanal. Chem. 2007, 388, 619-625.

[35] Jinno, K.; Ogawa, M.; Ueta, I.; Saito, Y. Trends Anal. Chem. 2007, 26, 27-35.

[36] Saito, Y.; Imaizumi, M.; Nakata, K.; Takeichi, T.; Kotera, K.; Wada, H.; Jinno, K. J. Microcol. Sep. 2001, 13, 259-264.

[37] Saito, Y.; Tahara, A.; Imaizumi, M.; Takeichi, T.; Wada, H.; Jinno, K. Anal. Chem. 2003, 75, 5525-5531.

[38] Saito, Y.; Tahara, A.; Ogawa, M.; Imaizumi, M.; Ban, K.; Wada, H.; Jinno, K. Anal. Sci. 2004, 20, 335-339.

[39] Saito, Y.; Ogawa, M.; Imaizumi, M.; Ban, K.; Abe, A.; Takeichi, T.; Wada, H.; Jinno, K. J. Chromatogr. Sci. 2005, 43, 536-541.

[40] Saito, Y.; Ogawa, M.; Imaizumi, M.; Ban, K.; Abe, A.; Takeichi, T.; Wada, H.; Jinno, K. Anal. Bioanal. Chem. 2005, 382, 825-829.

[41] Shirai, S.; Saito, Y.; Sakurai, Y.; Ueta, I.; Jinno, K. 
Anal. Sci. 2010, 26, 1011-1014.

[42] Li, P.; Xu, Z.; Yang, X.; Bi, W.; Xiao, D.; Choi, M. M. F. J. Chromatogr. A 2009, 1216, 3343-3348.

[43] Jinno, K.; Watanabe, H.; Saito, Y.; Takeichi, T. Electrophoresis 2001, 22, 3371-3376.

[44] Saito, Y.; Jinno, K. Chromatography 2001, 22, 151-158.

[45] Jinno, K.; Saito, Y.; Imaizumi, M. Bunseki Kagaku, 2001, 50, 775-783.

[46] Nelson, D. K.; Marcus, R. K. J. Chromatogr. Sci. 2003, $41,475-479$.

[47] Marcus, R. K.; Davis. W. C.; Knippel, B. C.; LaMotte, L.; Hill, T. A.; Perahia, D.; Jenkins, J. D. J. Chromatogr. A 2003, 986, 17-31

[48] Stanelle, R. D.; Sander, L. C.; Marcus, R. K. J. Chromatogr. A 2005, 1100, 68-75.

[49] Nelson, D. M.; Marcus, R. K. Anal. Chem. 2006, 78, 8462-8471.

[50] Stanelle, R. D.; Mignanelli, M.; Brown, P.; Marcus, R. K. Anal. Bioanal. Chem. 2006, 384, 250-258.

[51] Shirai, S.; Nakane, K.; Ueta, I.; Saito, Y. Chromatography 2011, 32, 127-133.

[52] Nakane, K.; Shirai, S.; Saito, Y.; Moriwake, Y.; Ueta, I.; Inoue, M.; Jinno, K. Anal. Sci. 2011, 27, 811-816.

[53] Abe, A.; Saito, Y.; Ueta, I.; Nakane, K.; Takeichi, T.; Jinno, K. J. Chromatogr. A 2009, 1216, 7456-7460.

[54] Tazawa, T.; Mori, Y.; Kobayashi, A.; Nakane, K.; Monobe, T.; Ueta, I.; Saito, Y. Anal. Sci. 2015, 31, 1137-1141.

[55] Inoue, M.; Nakazaki, H.; Tazawa, T.; Takeuchi, H.; Kobayashi, A.; Ueta, I.; Shirai, Y.; Moriuchi, K.; Saito, Y. Chromatography 2015, 36, 33-37.

[56] Wada, A. Sen'i Gakkaishi 1994, 50, 119-122. DOI: 10.2115/fiber.50.3_P119.

[57] McNair, H. M., Miller, J. M. Basic Gas Chromatography (2nd ed.), Wiley, Hoboken, NJ, 2009.

[58] Miller, J. M. Gas Chromatography, in Chromatography --Concepts and Contrasts-- (2nd ed.); Wiley-Inerscience, Hoboken, NJ, 2005; pp. 141-182 (Chapter 7). 